\title{
Morning sickness in pregnancy: mini review of possible causes with proposal for monitoring by diagnostic methods
}

\author{
Sophie Wylde ${ }^{1,3}$, Ezekiel Nwose ${ }^{2}$, Phillip Bwititi ${ }^{3}$
}

\author{
${ }^{1}$ School of Biomedical Sciences, Curtin University, Bentley, WA, Australia \\ ${ }^{2}$ School of Community Health, Charles Sturt University, Orange, NSW Australia \\ ${ }^{3}$ School of Biomedical Sciences, Charles Sturt University, Wagga Wagga, NSW, Australia
}

Received: 03 December 2015

Accepted: 07 January 2016

*Correspondence:

Sophie Wylde,

E-mail: sophie.wylde@curtin.edu.au

Copyright: (C) the author(s), publisher and licensee Medip Academy. This is an open-access article distributed under the terms of the Creative Commons Attribution Non-Commercial License, which permits unrestricted non-commercial use, distribution, and reproduction in any medium, provided the original work is properly cited.

\begin{abstract}
Nausea, vomiting in pregnancy (NVP) and hyperemesis gravidarum (HG) can have adverse effects on the mother and foetus. High risk groups for HG seem to include young women, non-smokers, non-Caucasian and women carrying multiple foetuses. Medical databases show that oestrogen, human chorionic gonadotrophin (hCG), thyroxine and insulin are associated with NVP and HG by mechanisms that are unclear. It is possible that NVP and HG may have different hormonal aetiologies. Other risk factors implicated in NVP and HG include psychological and genetic as well as external factors such as smoking status, age, bacterial infection and diet. The hypothesis hereby proposed is that hypoglycaemia, nausea and vomiting are associated with pregnancy perhaps due to disturbances in metabolic control and increased requirements for glucose by growing foetus. During overnight fasting, the resultant hypoglycaemia possibly triggers nausea and vomiting. This calls for investigations and of note is whether severities of NVP and HG correlate to blood glucose levels. This proposal reviews current thinking on the causes of NVP and HG as well as possible pathology evidence-based monitoring measures.
\end{abstract}

Keywords: Nausea, Vomiting, Pregnancy, Hyperemesis Gravidarum, Hormones

\section{INTRODUCTION}

Morning sickness is nausea and vomiting in pregnancy (NVP) with global incidence in up to $80 \%$ of pregnancies $^{1,2}$ and can be debilitating and affect daily functioning such as child care, social interactions, house chores, or employment. ${ }^{1,3,4}$ Morning sickness occurs at any time but is common in the morning ${ }^{5}$ and several theories exist on the causes and these include infections, hormonal control $^{6}$ and vitamin deficiency. ${ }^{5}$ Further information on the causes of NVP is required, particularly if triggers such as hypoglycaemia exist and the extent to which this may be associated with NVP.

The timing, frequency and duration of morning sickness vary between individuals and severe cases; hyperemesis gravidarum $(\mathrm{HG})$ are characterised by severe nausea and vomiting. ${ }^{7}$ This is seen in $1-3 \%$ of women presenting with NVP and can lead to hospitalisation, malnutrition, weight loss, vitamin deficiencies and low birth weights. $^{5,8,9}$ NVP and HG can initially be confused in early pregnancy since both are characterised by nausea and vomiting. However, slight differences exist, as indicated in Table 1.

NVP affects the mother and foetus and HG is concerning since it can lead to complications such as maternal and foetal morbidity, ${ }^{11}$ vitamin deficiencies, low birth weights as well as psychological impacts. ${ }^{5,12}$ Maternal mental health is often overshadowed by the symptoms of NVP and education to warn of nausea and vomiting and avoidance of certain foods are presented in pregnancy. This paper looks at the aetiology of NVP and HG as well as hypothesises and builds up on current knowledge on 
the causes and a framework for investigations is presented with a view to find possible relationships between risk factors and severities of NVP and HG. Such relationships may aid in predicting pregnancies at risk and also allow better management of these patients.

Table 1: Differential features of HG and NVP.

\begin{tabular}{|c|c|c|}
\hline Features & HG & NVP \\
\hline Prevalence & $\leq 3 \%$ & $\approx 80 \%$ \\
\hline Resolution & $\begin{array}{l}\text { Persistent } \\
\text { through } \\
\text { pregnancy }\end{array}$ & $\begin{array}{l}\text { Self-limiting at } \\
\text { about end of } 1^{\text {st }} \\
\text { trimester }\end{array}$ \\
\hline Risk factors & $\begin{array}{l}\text { Younger } \\
\text { women, multiple } \\
\text { foetuses }\end{array}$ & $\begin{array}{l}\text { None } \\
\text { remarkable }\end{array}$ \\
\hline Severity & More severe & Mild \\
\hline Hospitalisation & $\begin{array}{l}\text { Most likely and } \\
\text { multiple times }\end{array}$ & Less likely \\
\hline PUQE score ${ }^{10}$ & $\geq 13$ & $\leq 6$ \\
\hline
\end{tabular}

PUQE: Pregnancy-unique quantification of emesis and nausea

\section{Research question(s)/hypotheses}

The causes of NVP are not clear although increased production of HCG is the most popular factor other quantitatively testable metabolites e.g. blood glucose level, vitamin deficiencies are implicated. ${ }^{5,13}$ Several questions and hypotheses arise and these include:

i. If morning sickness is triggered by hypoglycaemia, then women with low blood glucose levels have a high frequency of morning sickness and the severity of NVP attack correlates with blood glucose level thus when there is no morning sickness, blood glucose level is normal.

ii. Morning sickness correlates with family history of morning sickness i.e. when there is weak family history of morning sickness attacks will be infrequent and less severe.

iii. Morning sickness correlates with spousal family history of morning sickness and when there is weak spousal family history of morning sickness attacks will be infrequent and less severe.

iv. Morning sickness correlates with foetal gender i.e. when the foetus is male/female then morning sickness attacks will be infrequent and less severe.

v. Morning sickness correlates with maternal hormonal levels such as oestrogens, progesterone, hCG, insulin. The levels of these hormones correlate with frequency and severity.

vi. Oxidative stress is a consequence of NVP or HG, equally oxidative stress triggers NVP and HG and magnitude of oxidative stress correlates with frequency and severity.

It is important to ascertain if low blood glucose levels triggers NVP and HG and this information is useful for management of morning sickness episodes as it may allow early intervention and indeed blood glucose levels can be measured by point of care testing at home. Studies need to be done in the first trimester using pre-screening questionnaires to gather information on the time of day and gravity of the incidents, age, weight, height, overall health (particularly diabetic status), dating of pregnancy and smoking/alcohol consumption pre-pregnancy, individual NVP family history and paternal NVP family history. These participants can be assigned a daily onehour window to self-measure blood glucose levels e.g. when NVP occurs. Equally participants also need to measure glucose at the same time during the days when they do not have morning sickness. This will be augmented by periodic measurements of hormones such as oestrogens, insulin and hCG as well as assessment of oxidative stress i.e. measurement of pro-oxidants and anti-oxidants. Other information that is necessary is food intake perhaps a few hours prior to the incident of morning sickness and with this, the severity of attacks recorded semi-quantitatively. Descriptive and inferential analyses can be performed on data collected during the pre-screening questionnaire and during the study. Data from the pre-screening questionnaire is useful to correlate frequency and severity of attacks with family history and spousal family history of morning sickness. Links between foetal gender and morning sickness incidents can be examined as well as external influences on frequency and severity of attacks such as alcohol consumption, smoking and exercise habits.

\section{DISCUSSION}

This mini-review paper looked at the current knowledge on NVP and HG in PubMed, EBSCOHost (Health) and Primo Search data bases in the last 30 years using 'nausea vomiting in pregnancy, hyperemesis gravidarum, hormones in pregnancy' as key words. The objective was to determine whether pathology-based evidence research reports have provided answers to these questions and the associated recommendations for laboratory-based management.

\section{Nausea and vomiting in pregnancy and hyperemesis gravidarum}

The risk factors for HG are young women, non-smokers, non-Caucasian women and those carrying multiple foetuses. ${ }^{14}$ Chan et al, noted that the timing of onset, duration and severity of symptoms differ among women and also among individual pregnancies in the same woman. ${ }^{15}$ Clinical presentations of $\mathrm{HG}$ are nausea, vomiting, enhanced sense of smell, food or fluid intolerance and lethargy but these can also present in a pregnant patient not suffering from HG. HG is concerning as it can lead to maternal and foetal morbidity, vitamin deficiencies, low birth weights and psychological impacts. ${ }^{5,11,12}$

NVP normally develops in the first trimester of pregnancy, peak around week 9 and usually subsides or resolves by the $12^{\text {th }}$ week ${ }^{2}$ and hormonal influences have 
been cited to contribute but it is not clear how hormones contribute to NVP or HG. ${ }^{6,11}$ Hormones associated with pregnancy and possibly NVP and HG include hCG, thyroxine, progesterone and oestrogen but psychological, genetic and external risk factors such as smoking, age, infection and diet are also implicated. ${ }^{2,16,17-22}$ Discretionally, these implicated causes are hereby briefly reviewed under five basic points:

- Endocrine: hormonal changes e.g. hCG, thyroxines, circadian rhythms

- Genetics: maternal and paternal contribution

- Psychological: conditioned or pre-conceived

- External: age, lifestyle, infections, nutrition

- Metabolic: hypoglycaemia

\section{Hormonal}

As mentioned the mechanism of hormonal control in NVP and HG is not clear but Verberg et al, suggested that high levels of oestrogen, hCG and progesterone were associated with severity of NVP. ${ }^{23}$ Huxley, suggested NVP to be caused by hCG and thyroxine, which are elevated in early pregnancy and Ismail and Kenny, proposed that hCG causes hyperemesis by stimulating secretory processes in the upper gastrointestinal tract. ${ }^{11,16}$ hCG has a positive correlation with $\mathrm{HG}$ and it is suggested that it influences the centre of the brain that controls nausea via thyroxine since hCG acts on maternal thyroid gland stimulating the release of thyroxines. ${ }^{24,25}$ In early pregnancy, hCG levels are high and TSH are low in blood and since hCG cross-reacts with TSH receptors stimulating the thyroid gland, increased hCG and/or thyroxine levels therefore influence severity and onset of nausea and emesis. ${ }^{16,26}$ Goodwin et al, reported severe vomiting in pregnant patients with high level of hCG and in thyroid stimulation and Niemeijer et al, confirmed a relationship between increased thyroid activity and HG. $^{24,27}$ It has been pointed out that suggestions of a relationship between hCG or thyroxine levels and NVP are possibly inaccurate since in cases of elevated hCG levels e.g. choriocarcinoma do not align with nausea and vomiting, equally some pregnant women with elevated hCG levels do not experience NVP and hyperthyroidism does not cause nausea or vomiting and patients with $\mathrm{HG}$ do not all develop hyperthyroidism. ${ }^{23}$

The effect of progesterone on NVP is not clear and it has been reported that in NVP patients, $40 \%$ had low and $40 \%$ had increased progesterone levels while in $20 \%$ there was no association. ${ }^{24}$ Studies have demonstrated a relationship between oestradiol levels and $\mathrm{NVP}^{28}$ and it has been noted that considering that physiological levels of oestrogens rise during the entirety of pregnancy, it is prudent to question why HG and NVP are most severe during the first trimester. ${ }^{23}$ An association between ACTH and cortisol via the HPA in HG has been proposed $^{29}$ and corticosteroids are used to treat nausea and vomiting in oncology patients. ${ }^{11}$ However, the use hydrocortisone and methylprednisolone in successful cessation of HG is not clear. ${ }^{8,29}$ It is important to ascertain the roles of these hormones in NVP and it is possible that a metabolic control concept involving various factors that are shown in Figure 1 is involved.

\section{Genetic}

Genetic factors are also implicated and studies have reported higher frequency of $\mathrm{HG}$ in New Zealand Pacific Island, United Kingdom Indian and Pakistani, Asian and African American women compared with ethnic European women and lower HG incidences in American Indian and Eskimo populations. ${ }^{23}$ Other studies have also confirmed ethnicity to correlate with severity and incidence of NVP. ${ }^{14,20}$ Differences have been reported in foetal genders and incidences of $\mathrm{HG}^{19,30}$ For instance, Fell et al, ${ }^{19}$ observed that foetal sex ratio among women who did not have an antepartum admission for hyperemesis was $51.2 \%$ males and $48.8 \%$ females and in women with 1-2 admissions for hyperemesis the proportions changed to $44.4 \%$ males and $55.6 \%$ females while in women with 3 or more admissions, the foetal sex distribution was $37.5 \%$ males compared with $62.5 \%$ females.

On familial link between incidence and severity of NVP, it was observed that women with sisters that experienced HG were 18 times likely to experience the same and those with $\mathrm{HG}, 33 \%$ had mothers that had severe NVP. ${ }^{20}$ Where data was accessible, the study also noted that in women with $\mathrm{HG}, 18 \%$ of their maternal grandmothers and $23 \%$ of paternal grandmothers also had been affected. Ismail and Kenny, noted that HG may decline in subsequent pregnancies if there is different paternity and suggested that with a different father, the incidence of HG may present with less severity or not at all. ${ }^{11}$ Due to paternal influence, a different father can potentially result in more severe HG. It is therefore possible that there are paternal and maternal influences on severity of NVP but mode is unknown and it is postulated that the genetic makeup of the foetus; maternal and paternal are involved.

In agreement with a maternal and sibling link in $\mathrm{HG}$, a study observed that $28 \%$ of women with HG had mothers that had also experienced NVP or HG, 19\% had sisters that had suffered from $\mathrm{HG}$ and those categorised as experiencing severe $\mathrm{HG}, 25 \%$ had symptomatic sisters. ${ }^{31}$ The study used an online survey on women possibly some without medical training who self-reported on their NVP or HG experience, thus there are limitations. Studies on female twin pregnancies have shown a strong correlation between identical twins whereby both suffer NVP compared to non-identical. ${ }^{32}$ Vikanes et al, reported that daughters were more likely to experience $\mathrm{HG}$ if their mother experienced HG in pregnancy and there was no relationship from paternal influence since fathers whose mothers experienced $\mathrm{HG}$ do not appear to influence $\mathrm{HG}$ in their children. ${ }^{33}$ As discussed, ethnicity of the mother correlates with $\mathrm{HG}$ and it was noted that the maternal 
genotype is more important than the foetal genotype in determining the risk of hyperemesis. ${ }^{14,20,34}$

\section{Psychological}

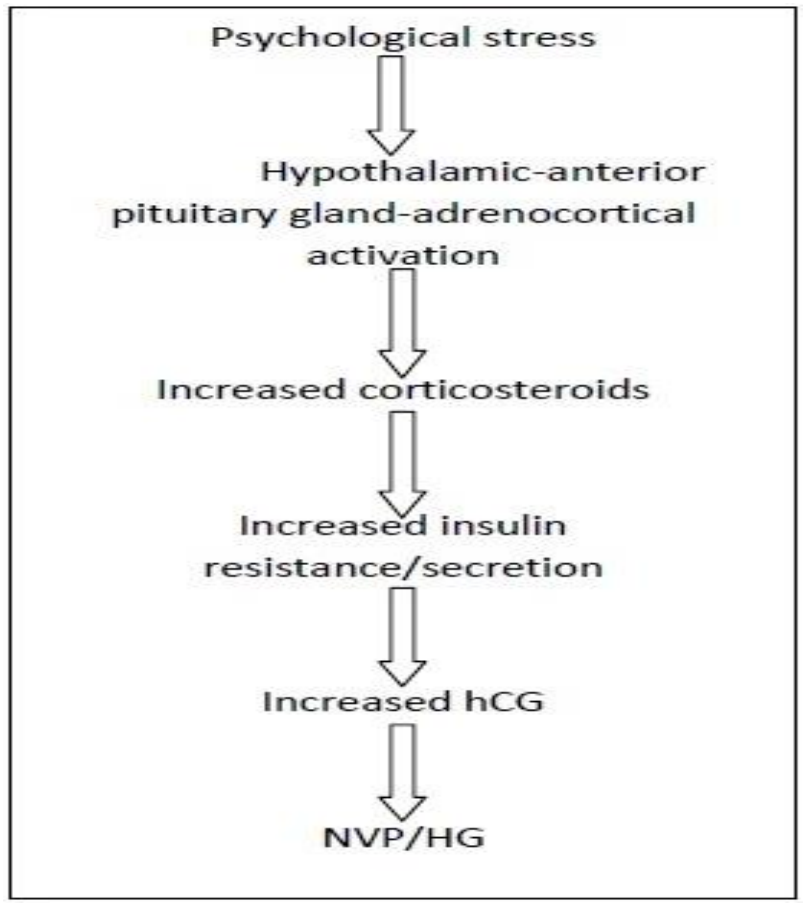

Figure 1: Potential implication of stress in complex metabolic control concept of NVP and HG.

Psychological factors influence the severity of NVP and Matteson et al, described 2 types; pre-conceived expectations and conditioning effect. In patients on chemotherapy, pre-conceived fear or anxiety or expectations of the treatment can induce nausea and vomiting. ${ }^{35}$ Conditioning occurs were administration of drugs are linked to nausea and vomiting and this conditioning possibly pre-empts nausea and vomiting. Such factors apply to pregnancy with expectation or previous experience of NVP inevitably inducing this. A further psychological influence on NVP was noted by Markl et al, who reported that lack of social support in the form of e.g. a housemate who does not necessarily have to be the father of the baby was associated with increases incidences of NVP. ${ }^{17}$ Further, the study reported that women that lived alone or who were not working had increased incidences of NVP. The authors proposed stress to be a predictor for developing NVP, as partners provide financial and emotional security, whereas being alone pre-disposes to vulnerability and therefore stress. We hypothesize that this is also involved in the complex metabolic control concept involving normal physiological feedback mechanisms as illustrated in Figure 1.

\section{External factors}

Smoking, age, infection and diet may influence the severity and incidence of NVP but surprisingly, smoking during pregnancy has been found to decrease the risk of HG possibly through interference with oestradiol metabolism. ${ }^{21,36}$ Passive smoking however does not appear to have the same effect and NVP incidences increase possibly due to different modes of action or due to pregnant women being more susceptible to irritants. ${ }^{37}$

Young women appear to have increased severity and incidence of $\mathrm{NVP}^{2}$ Weigel and Weigel, reported that women over 35 years had comparatively low rates of NVP while pregnant adolescents had high rates of NVP. ${ }^{38}$ It has been postulated that adolescent women had a stronger anabolic drive hence a poorer outcome of pregnancy than older women. ${ }^{39}$ This is possibly due to increased calorie consumption and increased levels of insulin and IGF-I, which deposit maternal stores at the expense of placental growth, ${ }^{16}$ perhaps contributing to NVP.

Helicobacter pylori infection correlates with $\mathrm{HG}$, perhaps due to changes in the $\mathrm{pH}$ of the gastrointestinal tract in addition to pregnancy-induced gastrointestinal tract dysmotility and altered immunity in pregnancy, all which favour $H$. pylori infection. ${ }^{6,22,40}$ Kuscu and Koyuncu, suggested that NVP beyond the second trimester are indicative of $H$. Pylori infection and other studies reported that the severity of NVP experienced correlated with the density of H. Pylori. ${ }^{29,40}$

Maternal diet, during pre-conception and in pregnancy is associated with the incidence and severity of NVP. Adequate sea-food, vegetables and water intake in prepregnancy reduce development of $\mathrm{HG}$ and it has been observed that women with NVP had diet high in calories, carbohydrates and added dietary sugars that came mostly from soft drinks but the study could not determine if consumption of such diet was to mitigate symptoms of NVP. ${ }^{2,18}$ Vitamin deficiency has been proposed as a casual factor for $\mathrm{HG}$ and it is possible that vitamin $\mathrm{B}$ deficiency may contribute, since the use of multivitamins containing vitamin B reduces the incidence of nausea and vomiting and it is pointed out that vitamin supplements taken in early pregnancy seems to protect against NVP. ${ }^{5,11}$ Niebyl and Goodwin, however, found no correlation between vitamin B and NVP but observations after administration of the vitamin showed a causal relationship since half of the patients stopped vomiting and most that had severe nausea reported diminution to mild or moderate nausea. ${ }^{41}$ Studies on vitamins $\mathrm{K}$ and thiamine suggest that low levels observed in HG patients by comparison with symptom-free women are a result of and not the cause of $\mathrm{HG}^{12,23}$ It must be pointed out that vomiting results in purging of dietary vitamins, whilst nausea decreases appetite and this turn results in dietary intake reduction thus lowering vitamin levels.

Physical health of mothers may have an evolutionary component contributing to the development of NVP. For 
example, NVP in early pregnancy was thought to be a mechanism to trigger awareness of pregnancy to the partner resulting in behaviour modification for protection and alerting the need for increased food supplies. ${ }^{42}$ These authors also proposed the notion of NVP as a mechanism for avoidance or purging of foods that may place the mother or foetus at risk. Caffeine and meat products are most cited to be adverse in pregnancy, with many women identifying the odours as a trigger for nausea. ${ }^{43}$

\section{Metabolic control in pregnancy}

Studies on NVP and HG in anorexic pregnant women are not clear since this can be super-imposed on anorexic behaviours but Magee et al, noted the importance of excluding anorexia in NVP. ${ }^{44}$ There are correlations between nausea and vomiting in hypoglycaemia and studies have shown that children presenting with hypoglycaemia also experience vomiting and following surgery, dexamethasone, which increases blood glucose level to mitigate nausea and vomiting can be administered. $^{45,46}$ NVP inevitably influences calorie intake with low calories decreasing insulin. Women with low BMI experience fewer symptoms of morning sickness. ${ }^{16}$ The mechanism underlying the reduction in nausea and vomiting in women with low BMI is reduced production of placental $\mathrm{hCG}$ and thyroxine as a result of reduced maternal insulin levels.

Figure 1, illustrates how psychological stress may exacerbate NVP and steroids are involved but the use of hydrocortisone and methylprednisolone on successful cessation of HG is not clear. ${ }^{8,29}$ The antiemetic properties of corticosteroid are possibly due to various factors acting in concert or individually and prostaglandin antagonism, ${ }^{47}$ stimulation of endorphins that elevate mood and appetite stimulation and reduction in tryptophan in neural tissue have been suggested. ${ }^{48,49}$

It can be postulated that in pregnancy, disturbances in metabolic control and increased requirements for glucose give rise to mild hypoglycaemia and during these situations, such as following overnight fasting; hypoglycaemia may trigger nausea and vomiting. The relationships between glycaemia and NVP and HG are not clear hence studies need to be carried out to assess glycaemia during NVP and HG as well as during periods that the women are not experiencing these conditions.

\section{Significance of finding biomarkers for nausea and vomiting in pregnancy and hyperemesis gravidarum}

It is important to understand the causes or mitigating factors for NVP and HG and possibly find biomarkers to predict or to monitor since the effects of NVP and HG are far reaching causing financial and social burdens. Glucose and hormonal measurements are within the capabilities of most laboratories and oxidative stress markers can also be measured and such assays need further studying especially their association and/or correlation with NVP and HG. Studies show that oxidative stress contributes to pathogenesis of various diseases including respiratory diseases and its contribution to NVP and HG needs research as well as the mitigation of anti-oxidants. ${ }^{50-52}$ These conditions affect the mother and the foetus and can lead to complications such as maternal and foetal morbidity, vitamin deficiencies, low birth weights as well as having psychological impacts, hence there is need for early identification and intervention. ${ }^{5,11,12}$ In a study on impact of NVP and daily functioning in pregnant women, O'Brien and Naber, reported that $83 \%$ responded that NVP affected their ability to perform normal daily activities and $34 \%$ of these had to change their daily schedules due to NVP. ${ }^{3}$ These are salient loss in productivity in everyday life, which translates to economic value.

\section{CONCLUSIONS}

NVP and HG affect maternal and foetal wellbeing but aetiology of these disorders is not clear and there is a case for hormonal cause, particularly hCG and oestrogen. NVP and HG perhaps have different control mechanisms with NVP possibly being an adjustment to a variety of hormonal changes that resolves when placental control commences while HG may be a consequence in individuals less tolerant to hormone changes. More studies on genetics are needed to elucidate familial and foetal influences. Other areas requiring studies are the roles of oxidative stress, vitamin deficiencies and importantly the role of glycaemic status as triggers of NVP episodes and also the potential role of insulin as a trigger. The complex relationship between NVP and influencing factors such as calorie intake/BMI, energy requirements, insulin and hCG requires further exploration. Whilst management options for NVP and $\mathrm{HG}$ are available, these have not been elaborated on in this review. Various antiemetic options and natural remedies are available to alleviate nausea and vomiting. Consideration must be given to maternal mental health as the effects of NVP and HG go beyond physical manifestation and influence the woman's ability to participate in daily routines and social interactions.

\section{Funding: No funding sources Conflict of interest: None declared Ethical approval: Not required}

\section{REFERENCES}

1. Koren G, Maltepe C. Pre-emptive therapy for severe nausea and vomiting of pregnancy and hyperemesis gravidarum. Journal of Obstetrics and Gynaecology. 2004;24(5):530-3.

2. Chortatos A, Haugen M, Iversen P, Vikanes A, Magnus P, Veierod M. Nausea and vomiting in pregnancy: associations with maternal gestational diet and lifestyle factors in the Norwegian Mother and Child Cohort Study. BJOG-An International 
Journal of Obstetrics and Gynaecology. 2013;120(13):1642-53.

3. O'Brien B, Naber S. Nausea and vomiting during pregnancy: effects on the quality of women's lives. Birth. 1992;19(3):138-43.

4. Lacasse A, Rey E, Ferreira E, Morin C, Berard A. Nausea and vomiting of pregnancy: what about quality of life? BJOG: International Journal of Obstetrics and Gynaecology. 2008;115(12):1484-93.

5. Niebyl J. Nausea and Vomiting in Pregnancy. The New England Journal of Medicine. 2010;363(16):1544-50.

6. Pirisi A. Meaning of morning sickness still unsettled. Lancet. 2001;357(9264):1272.

7. Vandraas K, Vikanes A, Stoer N, Troisi R, Stephasnsson O, Sorensen H. Hyperremesis gravidarum and risk of cancer in offspring, a Scandinavian registry-based nested case-control study. BioMed Central Cancer. 2015;15:398.

8. Yost N. A randomized, placebo-controlled trial of corticosteroids for hyperemesis due to pregnancy. Obstetrics and Gynaecology. 2003;102(6):1250-4.

9. Carpenter T. Is it morning sickness--or something worse? Registered Nurse. 2003;66(10):34-7.

10. Clark SM, Costantine MM, Hankins GD. Review of NVP and HG and early pharmacotherapeutic intervention. Obstetrics and Gynaecology International. 2012;20102:252676.

11. Ismail S, Kenny L. Review on hyperemesis gravidarum. Best Practice and Research Clinical Gastroenterology. 2007;21(5):755-69.

12. Toriello H, Tobiasz A, Erick M, Alessandri J, Bailey D, Brunetti-Pierri N. Maternal vitamin K deficient embryopathy: Association with hyperemesis gravidarum and Crohn disease. American Journal of Medical Genetics. 2013;161(3):417-29.

13. Maltepe C. Surviving morning sickness successfully: from patient's perception to rational management. Journal of Population Therapeutics and Clinical Pharmacology. 2014;21(3):e555-64.

14. Bailit J. Hyperemesis gravidarum: epidemiologic findings from a large cohort. American Journal of Obstetrics and Gynaecology. 2005;193(3):811-4.

15. Chan R, Olshan A, Savitz D, Herring A, Daniels J, Peterson $\mathrm{H}$, et al. Maternal influences on nausea and vomiting in early pregnancy. Maternal and Child Health Journal. 2011;15(1):122-7.

16. Huxley R. Nausea and vomiting in early pregnancy. Obstetrics and Gynaecology. 2000;95(5):779-82.

17. Markl G, Strunz-lehner C, Egen-lappe V, Lack N, Hasford J. The association of psychosocial factors with nausea and vomiting during pregnancy. Journal of Psychosomatic Obstetrics and Gynaecology. 2008;29(1):17-22.

18. Haugen M, Brantsater A, Meltzer H, Vikanes A, Magnus P, Grjibovski A. Diet before pregnancy and the risk of hyperemesis gravidarum. British Journal of Nutrition. 2011;106(4):596-602.

19. Fell D, Dodds L, Joseph K, Allen, V, Butler B. Risk factors for hyperemesis gravidarum requiring hospital admission during pregnancy. Obstetrics and Gynaecology. 2006;107(2):277-84.

20. Zhang Y, Cantor R, MacGibbon K, Romero R, Goodwin T, Mullin P, et al. Familial aggregation of hyperemesis gravidarum. American Journal of Obstetrics and Gynaecology. 2011;204(3):230(e1e7).

21. Vikanes A, Vangen S, Gunnes N, Samuelsen S, Magnus P, Grjibovski A. Maternal body composition, smoking, and hyperemesis gravidarum. Annals of Epidemiology. 2010;20(8): 592-8.

22. Mansour G, Nashaat E. Helicobacter pylori and hyperemesis gravidarum. International Journal of Gynaecology and Obstetrics. 2009;106(1):63-4.

23. Verberg, M, Gillott D, Al-Fardan N, Grudzinskas J. Hyperemesis gravidarum, a literature review. Human Reproduction Update. 2005;11(5):527-39.

24. Niemeijer M, Grooten I, Vos N, Bais, J, Painter R, van der Post J. Diagnostic markers for hyperemesis gravidarum: a systematic review and metaanalysis. American Journal of Obstetrics and Gynaecology. 2014;211(2):150.e1-e15.

25. Mori M, Amino N, Tamaki H, Miyai K, Tanizawa, O. Morning sickness and thyroid function in normal pregnancy. Obstetric Gynaecology. 1988;72:355-9.

26. Fantz C, Dagogo-Jack S, Ladenson J, Gronowski A. Thyroid function during pregnancy. Clinical Chemistry. 1999;45(12):2250-8.

27. Goodwin T, Montoro M, Mestman J, Pekary A, Hershman J. The role of chorionic gonadotropin in transient hyperthyroidism of hyperemesis gravidarum. Journal of Clinical Endocrinology and Metabolism. 1992;75(5):1333-7.

28. Lagiou P, Tamimi R, Mucci L, Trichopoulos D, Adami H, Hsieh C. Nausea and vomiting in pregnancy in relation to prolactin, estrogens, and progesterone: a prospective study. Obstetrics and Gynaecology. 2003;101(4):639-44.

29. Kuscu N, Koyuncu F. Hyperemesis gravidarum: current concepts and management. Postgraduate Medical Journal. 2002;78(916):76-9.

30. Schiff M, Reed S, Daling J. The sex ratio of pregnancies complicated by hyperemesis gravidarum. Paediatric \& Perinatal Epidemiology. 2001;15(4):A30.

31. Fejzo M, Ingles S, Wilson $\mathbf{M}$, Wang W, Macgibbon K, Romero R, Goodwin T. High prevalence of severe nausea and vomiting of pregnancy and hyperemesis gravidarum among relatives of affected individuals. European Journal of Obstetrics, Gynaecology and Reproductive Biology. 2008;141(1):13-7.

32. Corey L, Berg K, Solaas M, Nance W. The epidemiology of pregnancy complications and outcome in a Norwegian twin population. Obstetrics and Gynaecology. 1992;80(6):989-94.

33. Vikanes A, Skjærven R, Grjibovski A, Gunnes N, Vangen S, Magnus P. Recurrence of hyperemesis gravidarum across generations: population based cohort study. British Medical Journal. 2010;340(7755):1071-5. 
34. Nelson-Piercy C. Recurrence of hyperemesis across generations. British Medical Journal. 2010;340(7755):1043-4.

35. Matteson S, Roscoe J, Hickok J, Morrow G. The role of behavioral conditioning in the development of nausea. American Journal of Obstetrics and Gynaecology. 2002;186(5):S239-43.

36. Baron J, Lavecchia C, Levi F. The antiestrogenic effect of cigarette smoking in women. American Journal of Obstetrics and Gynaecology. 1990;162(2):502-14.

37. Zhang J, Cai W. Severe vomiting during pregnancy: Antenatal correlates and fetal outcomes. Epidemiology. 1991;2(6):454-7.

38. Weigel M, Weigel $\mathrm{R}$. The association of reproductive history, demographic factors, and alcohol and tobacco consumption with the risk of developing nausea and vomiting in early pregnancy. American Journal of Epidemiology. 1988;127(3):562-70.

39. Coad, J, Al-rasasi B, Morgan J. Nutrient insult in early pregnancy. Proceedings of the Nutrition Society. 2002;1(1):51-9.

40. Bagis, T, Gumurdulu Y, Kayaselcuk F, Yilmaz, E, Killicadag E, Tarim E. Endoscopy in hyperemesis gravidarum and Helicobacter pylori infection. International Journal of Gynaecology and Obstetrics. 2002;79(2):105-9.

41. Niebyl J, Goodwin T. Overview of nausea and vomiting of pregnancy with an emphasis on vitamins and ginger. American Journal of Obstetrics and Gynaecology. 2002;186(5): S253-5.

42. Flaxman S, Sherman P. Morning sickness: A mechanism for protecting mother and embryo. Quarterly Review of Biology. 2000;75(2):113-48.

43. Lawson C, LeMasters G, Wilson K. Changes in caffeine consumption as a signal of pregnancy. Reproductive Toxicology. 2004;18(5):625-33.

44. Magee L, Shrim A, Koren G. Diagnosis and Management of nausea and vomiting in pregnancy.
Fetal and Maternal Medicine Review. 2006;17(1):4567.

45. Josefson J, Zimmerman D. Hypoglycemia in the Emergency Department. Clinical Pediatric Emergency Medicine. 2009;10(4): 285-91.

46. Dhatariya K. Does dexamethasone-induced hyperglycaemia contribute to postoperative morbidity and mortality? British Journal of Anaesthesia. 2013;110(5):674-5.

47. Rich MW, Abdulhayaglu G, Di Saia PJ. Methylaprednisolone as antiemetic during cancer chemotherapy-a pilot study. Gynaecologic Oncology. 1980;9(2):193-8.

48. Harris AL. Cytotocytic-therapy-induced vomiting is mediated via enkephalin pathways. Lancet. 1982;1(8274):714-6.

49. Young S. Mechanism of decline in rat brain 5hydroxytryptamine after induction of liver tryptophan pyrrolase by hydrocortisone: roles of tryptophane catabolism and kynurenine synthesis. British Journal of Pharmacology. 1981;74(3):695700.

50. Birben E, Sahiner UM, Sackesen C, Erzurum S, Katayci O. Oxidative stress and antioxidant defense. World Allergy Organ Journal. 2012;5(1):9-19.

51. Komaravelli N, Casola A. Respiratory viral infections and subversion of cellular antioxidant defences. Journal of Pharmacogenomics Pharmacoproteonomics. 2014;5(4):ppii: 1000141.

52. Komaravelli N, Kelley JP, Garofalo MP, Wu H, Casola A, Kolli D. Role of dietary antioxidants in human metapneumovirus infection. Virus Research. 2015;200:19-23.

Cite this article as: Wylde S, Nwose E, Bwititi P. Morning sickness in pregnancy: mini review of possible causes with proposal for monitoring by diagnostic methods. Int J Reprod Contracept Obstet Gynecol 2016;5:261-7. 